\title{
Promoção da saúde através de parcerias para a pesquisa na América Latina*
}

\author{
Jaime Miranda1,2, Ana Cristina Castro-Ávila ${ }^{3,4}$ e Luis Alejandro Salicrup ${ }^{5,6}$
}

\begin{abstract}
Jaime Miranda e cols. argumentam que as parcerias são fundamentais para estabelecer e sustentar as capacidades de pesquisa em saúde na América Latina
\end{abstract}

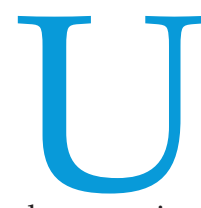

$\mathrm{m}$ dos principais objetivos da política de pesquisa para a saúde da Organização Pan-Americana da Saúde (OPAS) ${ }^{1}$ é o desenvolvimento de parcerias para implementar estratégias de saúde adequadas e sustentáveis. A política visa facilitar o desenvolvimento e a ampliação de produtos e tecnologias econômicos e acessíveis que sejam relevantes para as necessidades de saúde pública nas Américas, estabelecer grupos de pesquisa nos Estados Membros da OPAS com competências que permitam o intercâmbio de conhecimentos e facilitar a colaboração entre as diversas partes relevantes - incluindo os setores público, governamental, acadêmico e privado para abordar as prioridades nacionais e regionais. ${ }^{1}$

As primeiras tentativas de estabelecer parcerias na região ocorreram na década de 1970. Por exemplo, a UNESCO, o Programa das Nações Unidas para o Desenvolvimento e várias outras agências da ONU financiaram parcerias sul-sul entre instituições da América Latina com o objetivo de apoiar redes de pesquisa em biologia, microbiologia e ciências biomédicas. $^{2} \mathrm{O}$ objetivo era promover a troca de conhecimentos e estabelecer capacidades de pesquisa em países com infraestrutura de pesquisa menos estabelecida, formando os seus cientistas em instituições maiores e mais experientes em países como Argentina, Brasil e México. No entanto, muitas redes se desfizeram devido à falta de financiamento nacional. Décadas mais tarde, estão surgindo exemplos notáveis de colaboração e parcerias norte-sul e sul-sul.

\section{MENSAGENS-CHAVE}

- As parcerias têm sido e continuarão a ser cruciais para o avanço da pesquisa em saúde de alta qualidade na América Latina e em todo o mundo.

- A política de pesquisa para a saúde da OPAS serve como referência na América Latina e promove um forte apoio à formação de parcerias.

- As parcerias para a pesquisa na região devem ocorrer entre partes iguais que compartilham objetivos e metas comuns.

- Para que as parcerias sejam eficazes, as partes envolvidas precisam estabelecer uma visão e missão comuns, negociar papéis, responsabilidades e ações, definir objetivos e estabelecer processos para a negociação e a partilha de poder.

- Nem todas as parcerias são idênticas, devendo evoluir com base em suas potencialidades únicas.

- AAmérica Latina precisará se adaptar a um mundo em mudança compartilhando informações. As iniciativas de agrupamento de dados representarão um desafio para os paradigmas de colaboração existentes, e um referencial estruturado para as parcerias pode ajudar a definir um caminho que permita o avanço na região.

\footnotetext{
* Tradução oficial para o português realizada pela Organização Pan-Americana da Saúde. Em caso de discrepância, prevalecerá a versão original em inglês.

1 Pesquisador Faculdade de Medicina, Universidade Peruana Cayetano Heredia, Avenida Honorio Delgado 430, San Martin de Porres, Lima 31, Peru. $₫$ Jaime.Miranda@upch.pe
}

\footnotetext{
5 Assessor sênior para pesquisa em saúde global, Organização Pan-Americana da Saúde/Organização Mundial da Saúde, Washington, DC

20037-2895, EUA
Assessor sênior para pesquisa em saúde global, Centro para a Saúde Global, Instituto Nacional do Câncer, Institutos Nacionais de Saúde dos EUA, Bethesda, Maryland, EUA
}

Doenças Crô tano Heredia, Miraflores, Lima 18, Peru

Pesquisadora Departamento de Ciências da Saúde, Universidade de York, York, Yorkshire YO10 5DD, Reino Unido

Pesquisadora Curso de Fisioterapia, Faculdade de Medicina, Universidade do Desenvolvimento, Clínica Alemana, Santiago 7610658, Chile 
Os termos parceria e colaboração muitas vezes são usados como sinônimos, mas representam relações diferentes. ${ }^{3}$ Uma colaboração envolve partes que trabalham juntas em um empreendimento intelectual, enquanto que uma parceria significa uma relação entre iguais que possuem objetivos e metas comuns, definidos em conjunto. ${ }^{3}$

O desenvolvimento, implementação e manutenção de parcerias pode ser complexo, exigindo a compreensão dos fatores que as facilitam ou dificultam. As barreiras culturais e os campos de conhecimento extremamente compartimentalizados também podem afetar o estabelecimento de parcerias significativas. Exploramos aqui algumas das características das parcerias de pesquisa existentes que conseguem maximizar as oportunidades de financiamento e compartilhar recursos, metas e objetivos na América Latina. Selecionamos estudos de caso para destacar interações interdisciplinares, interagências, norte-sul, sul-sul e entre a academia e a indústria.

\section{PARCERIAS PARA GERAR NOVOS CONHECIMENTOS}

O National Heart, Lung, and Blood Institute e o United Health Group identificaram a necessidade premente de estabelecer capacidades de pesquisa e infraestrutura para enfrentar o grande desafio da carga crescente de doenças não transmissíveis em países de renda baixa e média. ${ }^{4}$ Juntos, criaram a Iniciativa para Doenças Crônicas, implementada entre 2009 e 2014, para apoiar uma rede de 11 centros de pesquisa em 10 países de renda baixa e média, quatro deles na América Latina (México, Guatemala, Peru e Argentina). ${ }^{4}$ Cada centro de excelência se associou a pelo menos uma instituição acadêmica de alta renda nos Estados Unidos, Canadá, Austrália ou Europa e a uma organização local, que poderia ser uma universidade ou instituição voltada à pesquisa ou à saúde. ${ }^{4}$ Os países de renda baixa e média foram os principais beneficiários das subvenções concedidas pelo programa, o que os obrigou a desenvolver rapidamente as suas instituições científicas e a melhorar os seus procedimentos administrativos e de gestão de financiamento para que atendessem aos padrões internacionais. ${ }^{4}$

Em 2016, os quatro centros de excelência na região da América Latina já haviam participado de 14 projetos de pesquisa colaborativos, com diversos desenhos experimentais, produzindo 86 publicações e 97 apresentações de pesquisa e formando 216 pesquisadores em cursos de pós-graduação. ${ }^{4}$ Além disso, essa parceria facilitou um processo de harmonização de dados que permitiu a exploração de novas questões de pesquisa voltadas a doenças não transmissíveis em centros de excelência na região.

Os centros de excelência na América Latina e os seus parceiros globais se beneficiaram mutuamente do programa, não só através dos seus avanços científicos, como também da interação ao nível pessoal e institucional ao longo de todo o processo, desde a concepção dos estudos até a divulgação dos seus resultados. Os centros de excelência para doenças crônicas não transmissíveis conseguiram fortalecer a sua capacidade de pesquisa, compartilhar dados e redes, promover a agregação do ganho e do mérito e transformar uma única iniciativa de financiamento em um empreendimento de longo prazo. Infelizmente, sem financiamento sustentado, as interações em andamento podem enfrentar dificuldades, colocando em risco a parceria e as suas atividades de pesquisa. Isto enfatiza a importância de apoiar as redes que trabalham em consonância com as atuais necessidades de pesquisa e estudam a saúde a partir de uma perspectiva multidisciplinar e de multimorbidade, em vez de trabalhar de forma compartimentalizada. ${ }^{5}$

\section{PARCERIAS QUE PROMOVEM O ENVOLVIMENTO DAS PARTES RELEVANTES}

Os pesquisadores da região raramente têm a oportunidade e o apoio dos governos para testar e recomendar intervenções de saúde pública. O Projeto GUIA (Guia Útil para Intervenções de Atividade Física no Brasil e na América Latina) é uma parceria de pesquisa multidisciplinar internacional que adotou uma abordagem diferente. Ao invés de avaliar novas intervenções por meio de estudos clínicos randomizados e, em seguida, ampliar as intervenções mais bem sucedidas, o projeto avalia intervenções de saúde pública que já estão sendo implementadas na América Latina.

Utilizando um processo iterativo de avaliação, o agrupamento de evidências e a adaptação de intervenções, o Projeto GUIA fez estudos acadêmicos para avaliar diferentes programas de saúde pública, o que levou à ampliação em escala federal, no Brasil, de programas que oferecem aulas de atividade física na comunidade (Academia da Cidade) e programas de incentivo à atividade física pela oferta de recursos e espaços públicos (Academia da Saúde). Programas semelhantes em outros países, como Colômbia e Chile, também estão sendo avaliados e ampliados. ${ }^{6}$

O Projeto GUIA foi financiado e coordenado pelos Centros para o Controle e Prevenção de Doenças dos EUA e pelo Centro de Pesquisa sobre Prevenção de Saint Louis, EUA, em parceria com três universidades brasileiras. ${ }^{6} \mathrm{O}$ projeto utilizou as formas de colaboração entre instituições de saúde pública e órgãos governamentais que já existiam antes do início do projeto. Uma rede diversificada de parceiros do meio acadêmico, do governo (em todos os níveis) e de instituições não-governamentais (como a OPAS e o CELAFISC [Centro de Estudos do Laboratório de Aptidão Física de São Caetano do Sul]) foram convidados a participar do projeto. Um elemento importante para o êxito do projeto foi a sua sensibilidade cultural, que permitiu aos parceiros identificar ou priorizar os esforços de pesquisa de acordo com o contexto local. ${ }^{6}$

OProjeto GUIA exemplifica a importância da interação com as partes relevantes desde o início, permitindo que os pesquisadores e formuladores de políticas usem e gerem evidências fundamentadas no trabalho prático. A divulgação dos resultados para serem usados por entidades regionais e a sua implementação dentro dos programas foram reconhecidas como aspectos importantes da parceria. ${ }^{6}$

As reflexões sobre o sucesso do Projeto GUIA também destacaram a importância do momento da implementação. O projeto começou em uma ocasião em que as agências de saúde pública brasileiras estavam prontas para adotar iniciativas de prevenção de doenças não transmissíveis $^{6}$ e em que estava surgindo um grupo de jovens pesquisadores talentosos na América Latina, ${ }^{7}$ o que mostra que o investimento em pesquisadores logo no início de suas carreiras pode evoluir para iniciativas maiores. No entanto, a retenção de talentos requer mais investimento. Sem esse apoio, será difícil manter o fluxo de talentos para trabalhar, inovar e promover as iniciativas de saúde pública destinadas a combater as doenças não 
transmissíveis na região. É fundamental contar com uma massa crítica de talentos, usando a sua experiência para promover as lições aprendidas ou manter os conhecimentos e o engajamento nas políticas em vários níveis.

\section{PARCERIAS PARA TECNOLOGIAS MAIS APROPRIADAS E ECONÔMICAS}

A transferência de inovações de uma região para outra não resulta necessariamente nos resultados desejados, e o desenvolvimento de novas tecnologias médicas custa tempo e dinheiro. Por isso, as parcerias são cruciais para a implementação de tecnologias médicas apropriadas e econômicas na América Latina. Parcerias entre a academia e a indústria, incluindo a colaboração norte-sul e sul-sul, podem levar a intervenções com melhor relação custo-eficácia, apropriadas, econômicas e sustentáveis para enfrentar os principais desafios de saúde pública na região. Além disso, ao envolverem diversas entidades dentro de instituições e entre diferentes instituições, essas parcerias podem ajudar a estabelecer a capacidade de pesquisa interdisciplinar e formar pesquisadores que se envolvam no trabalho de pesquisa, desenvolvimento e inovação na América Latina.

Um exemplo de promoção de parcerias na região para a inovação na pesquisa em saúde é o financiamento competitivo oferecido pelos Institutos Nacionais de Saúde dos EUA, por meio do seu Instituto Nacional do Câncer. Essas parcerias são especialmente relevantes dada a carga de doença na região: $85 \%$ dos cânceres de colo do útero em todo o mundo ocorrem em países de renda baixa e média, e 16\% dos casos ocorrem nas Américas. ${ }^{8}$ Uma parceria atual apoiada pelos Institutos Nacionais de Saúde dos EUA/ Instituto Nacional do Câncer envolve instituições públicas e privadas na América Latina e nos Estados Unidos que estudam o uso acessível e econômico de diagnósticos moleculares para a detecção precoce de subtipos do vírus do papiloma humano (HPV) que podem levar ao câncer de colo do útero. Especificamente, pesquisadores da América Latina e da Agência Internacional de Pesquisa em Câncer estão trabalhando para desenvolver um teste de HPV baseado na detecção da atividade da oncoproteína E6/E7, que está presente nos oito subtipos mais comuns de HPV associados ao câncer de colo do útero. $\mathrm{O}$ estudo utilizará redes de pesquisa clínica pré-existentes na América Latina para avaliar o potencial do teste como uma ferramenta de rastreamento padrão para o câncer de colo do útero. ${ }^{9}$ Outro exemplo de parceria apoiada por este programa, que se baseou em redes já existentes na América Latina, é o da transformação de um dispositivo ablativo, o CryoPen(R) Cryosurgical System, em um dispositivo de crioterapia barato e portátil que pode ser operado por qualquer profissional da saúde. ${ }^{10}$

As parcerias entre a indústria, o meio acadêmico e o governo, descritas na política de pesquisa para a saúde, dependem da definição de uma agenda conjunta que esclareça as responsabilidades, fortaleça as capacidades e promova o compartilhamento de dados e redes, para ter um impacto duradouro sobre as necessidades atuais e emergentes de saúde pública. No entanto, essas parcerias podem ser enfraquecidas se as diferentes agências não conseguirem alinhar as suas prioridades, e mudanças nas agendas políticas podem criar obstáculos. O desenvolvimento dessas parcerias depende das oportunidades de financiamento existentes e futuras. A menos que os parceiros envolvidos contem com um plano sustentado de desenvolvimento de produtos, existe o risco de que, quando o financiamento terminar, as interações facilitadas pelas parcerias sejam interrompidas, colocando em risco o processo de pesquisa e inovação e, assim, os resultados pretendidos.

\section{DISCUSSÃO}

As parcerias multidisciplinares promovem um maior entendimento entre as partes relevantes dos setores público e privado, tanto a nível nacional como internacional. Elas são fundamentais para enfrentar os desafios atuais e futuros da saúde: o tratamento de doenças transmissíveis e não transmissíveis, a manutenção das intervenções de saúde pública e a criação de soluções inovadoras para problemas comuns, que possam ser aplicadas em países de renda baixa e média.

Para que as parcerias sejam eficazes, as partes relevantes precisam estabelecer uma visão e uma missão comum, negociar papéis, responsabilidades e ações, definir objetivos e estabelecer processos para a negociação e a partilha de poder. ${ }^{11}$ Será necessário criar alianças estratégicas com todas as partes potencialmente envolvidas (governos, universidades, setor privado, financiadores, organizações internacionais, sociedades profissionais, organizações comunitárias e grupos de pacientes) para abordar as prioridades nacionais através da pesquisa em saúde. As parcerias entre diferentes atores também são cruciais para alcançar os objetivos da política de pesquisa para a saúde: melhorar a equidade, a saúde e o desenvolvimento. ${ }^{12}$

As novas parcerias de pesquisa muitas vezes fracassam devido a orçamentos irreais e à falta de lideranças, processos de negociação, trabalho em equipe e competências gerenciais. Atualmente, os pesquisadores da América Latina adquirem essas competências durante o trabalho prático, nas fases mais avançadas de sua carreira. A introdução e o desenvolvimento dessas habilidades durante os cursos de graduação e pós-graduação podem promover relacionamentos duradouros e sustentáveis entre iguais, que são um pré-requisito para o estabelecimento de parcerias significativas. A incorporação dessas competências no currículo exigirá uma outra parceria: entre instituições acadêmicas e governos para a capacitação.

A região da América Latina pode tirar proveito de sua população diversificada e de seus idiomas comuns, principalmente espanhol e português, para estabelecer parcerias que, de outra forma, provavelmente não funcionariam. Será necessário um trabalho de adaptação, pois os desafios existentes (tais como a agenda inacabada para as doenças infecciosas, o fortalecimento dos sistemas de saúde e a promoção da cobertura universal de saúde) serão combinados a desafios emergentes, como a multimorbidade, as doenças não transmissíveis e as abordagens de pesquisa ligadas à utilização de grandes volumes de dados (big data) - questões que serão melhor abordadas por meio de parcerias bem sucedidas. ${ }^{13}$ A política de pesquisa para a saúde da OPAS serve como base para envolver e alinhar várias agências dedicadas a um objetivo comum: a pesquisa para a saúde e a equidade. Essas alianças e os seus resultados serão cruciais para fortalecer os sistemas de saúde que enfrentam os grandes desafios de saúde pública. Também permitirão comparar os avanços no setor da saúde, de modo que os países possam formular planos baseados em evidências para melhorar a qualidade global e o acesso aos serviços 
de saúde - que constituem importantes pilares da estratégia para o acesso universal à saúde que a OPAS e todos os Estados Membros se comprometeram a implementar.

Agradecimentos. Agradecemos a Luis Gabriel Cuervo por suas ideias e sugestões para este artigo e a Miguel Ossandon por compartilhar informações valiosas sobre o escopo do NCI Program on Affordable Cancer Technologies for LMIC. Também agradecemos a Samantha Brew pela ajuda na edição das primeiras versões deste manuscrito. A Ana Amelia Correa pela revisão da tradução.

Contribuição dos autores. JJM concebeu a ideia e a estrutura deste artigo e escreveu a primeira versão, com o apoio de ACCA e de LAS. ACCA realizou a primeira revisão da literatura, contribuiu para a redação do manuscrito e comentou todas as versões preliminares e revisões. Todos os autores contribuíram com conteúdo intelectual, editaram o manuscrito e aprovaram a versão final.

Conflito de interesses. Lemos e compreendemos a política do BMJ sobre a declaração de interesses e declaramos os seguintes interesses: JJM é membro do Comitê Assessor sobre Pesquisa em Saúde (ACHR) da OPAS desde setembro de 2014 e presidiu o comitê de setembro de 2014 a abril de 2016, além de atuar como pesquisador-chefe no Centro Peruano de Excelência financiado pelo NHLBI. LAS trabalhou no NCI/NIH e na OPAS/OMS.

Procedência do artigo e revisão por pares. Encomendado; não submetido à revisão por pares externa.

\section{REFERÊNCIAS}

1. Organização Pan-Americana da Saúde. Policy on research for health (CD49/10). PAHO/WHO, 2009. http://www1.paho .org/hq/dmdocuments / 2010/Policy ResearchHealthPOR.pdf

2. Sobre RELAB. Red Latinoamericana de Ciencias Biologicas. http://relab.biologia .ucr.ac.cr / ?pageid $=10$

3. Glazer G, Alexandre C, Reid Ponte P. Legislative: Partnership or collaboration: words matter. Online Journal of Issues in Nursing 2008;13. https://www.nursingworld.org /MainMenuCategories/ANAMarketplace /ANAPeriodicals/OJIN/TableofContents /vol132008/No2May08/PartnershiporCollaboration.html

4. Engelgau MM, Sampson UK, Rabadan-Diehl C, et al, National Heart, Lung, and Blood Institute-United Health Global Health Centers of Excellence Collaborators. Tackling NCD in LMIC: achievements and lessons learned from the NHLBI-UnitedHealth Global Health Centers of Excellence Program. lob Heart 2016;11:515. doi:10.1016/j. gheart.2015.12.016

5. Academy of Medical Sciences. Addressing the global challenge of multimorbidity: Lessons From BRICS countries. Academy of
Medical Sciences, 2017. https://acmedsci .ac.uk/ file-download/3855485

6. Parra DC, Hoehner CM, Hallal PC, et al. Scaling up of physical activity interventions in Brazil: how partnerships and research evidence contributed to policy action. Glob Health Promot 2013;20:5-12. doi:10.1177/1757975913502368

7. Pratt M, Brownson RC, Ramos LR, et al. Project GUIA: A model for understanding and promoting physical activity in Brazil and Latin America. J Phys Act Health 2010;7(Suppl 2):S131-4. doi:10.1123/ jpah .7.s2.s131

8. International Agency for Research on Cancer. GLOBOCAN 2012 cervical cancer: Estimated incidence, mortality and prevalence worldwide in 2012. http://globocan iarc.fr/old/FactSheets / cancers / cervix -new.asp

9. National Cancer Institute. Supporting the development of affordable technologies for cancer detection, diagnosis, and treatment in LMICs. 2016. https://www.cancer.gov /about-nci/organization/cgh/blog/2016

10. NIH RePORTER. CryoPen: an innovative treatment for cervical precancer in low-resource setting. NIH Research Portfolio
Declaração. As opiniões externadas neste manuscrito são de responsabilidade do(s) autor(es) e não refletem necessariamente os critérios e políticas da RPSP/ PAJPH e/ou da OPAS/OMS.

Série Fortalecimiento da pesquisa em saúde nas Américas. Este artigo faz parte de uma série proposta pela OPAS e encomendada pelo BMJ, o qual foi responsável pela revisão por pares, edição e decisão de publicar o artigo, sem participação da OPAS. As despesas referentes à publicação desta série em Open Access foram financiadas pela OPAS. Para ler os outros artigos da série em inglês, visite www.bmj.com/health-research-americas; acesso à série em espanhol e português em https://www.paho .org/journal/es/numeros-especiales / fortalecimiento-investigacion-para -salud-americas
Online Reporting Tools. https://project reporter.nih.gov/project_info_description .cfm?aid $=9355105 \&$ icde $=37212702$

11. Gallant MH, Beaulieu MC, Carnevale FA. Partnership: an analysis of the concept within the nurse-client relationship. J Adv Nurs 2002;40:149-57. doi:10.1046/j.1365-2648.2002.02357.x

12. Pan American Health Organization/ World Health Organization. Milestones for a Policy on Research for Health. 2014 https://www.paho.org/hq/index. php ?Itemid $=41201$

13. Sterman JD. Learning from evidence in a complex world. Am J Public Health 2006;96:505-14. doi:10.2105 / AJPH.2005.066043

Como citar (artigo original): BMJ 2018;362:k2690 http:/ / dx.doi.org/10.1136/bmj.k2690

Manuscrito original em inglês publicado em 16 de julho de 2018. 